\title{
Kawasaki disease, Epstein-Barr virus and coronary artery aneurysms
}

\author{
G A Culora, I E Moore
}

\begin{abstract}
Aim-To establish whether infection with Epstein-Barr virus (EBV) contributed to the development of coronary artery lesions in a six year old child with an aneurysm and stenoses of the coronary arteries and suspected Kawasaki disease. Methods-Postmortem paraffin wax sections of the coronary artery and myocardium were examined by in situ hybridisation for expression of EBER-1 (EBV-encoded RNA-1). Positive controls consisted of an EBV positive case of Hodgkin disease and a case of posttransplantation lymphoma.

Results-No EBER-1 positive cells were identified in either myocardium or walls of the coronary artery.

Conclusions-Although EBV has been implicated in the aetiology of Kawasaki disease and development of coronary artery lesions, this process was not confirmed in this patient. It is likely that an unusual immunological reaction to a primary EBV infection with suspected deregulation of $T$ helper cell activity leads to severe coronary artery damage in early childhood.

(F Clin Pathol 1997;50:161-163)
\end{abstract}

Keywords: Kawasaki disease; Epstein-Barr virus.

Kawasaki disease is a multisystem acute vasculitis, which classically affects children between six months and five years of age. It has an incidence in the United Kingdom of 1.5 per $100000 .^{1}$ Diagnosis is based on strict clinical criteria which include fever lasting five or more days, conjunctivitis, inflammation of the mucous membranes of the mouth and pharynx, oedema, erythema and desquamation of the skin of the hands and feet, a polymorphous rash, and cervical lymphadenopathy. There are no specific laboratory tests but a raised erythrocyte sedimentation rate and thrombocytosis may be present. Staphylococcal and streptococcal infections, measles, leptospirosis, and rickettsial disease have to be excluded when the diagnosis is made. The disease is usually self-limiting but $15-25 \%$ of patients may develop coronary artery abnormalities, and up to $2 \%$ of these patients subsequently die. ${ }^{2}{ }^{3}$ The regression of aneurysmal changes has been shown to be related to the severity of coronary artery lesions and initial treatment with aspirin and immunoglobulin. ${ }^{45}$ Death has been reported up to 14 years after the acute illness. ${ }^{6}$
Epidemiological evidence strongly suggests that an infectious agent is involved in the aetiology of Kawasaki disease. Several organisms have been implicated. Initially rickettsia-like bodies were detected on electron microscopy of lymph nodes from patients with Kawasaki disease, but other studies failed to confirm these findings. ${ }^{78}$ House dust mite was also suggested as a possible cause (but this was not substantiated), as were leptospires, Propionibacterium acnes and several viruses, including adenovirus, herpes simplex, enteroviruses, and EpsteinBarr virus (EBV). ${ }^{9}$ However, in many cases, the serological evidence of a viral infection has not been documented and more sophisticated methods such as detection of reverse transcriptase activity are required to detect the presence of some of the viruses (retroviruses)..$^{10}$

A number of $\mathrm{T}$ - and B-cell abnormalities have been reported in the acute phase of Kawasaki disease. These include deficient T8 positive suppressor/cytotoxic $T$ cells, increased T4 positive activated helper $\mathrm{T}$ cells bearing HLA-DR surface antigens, and large numbers of B cells spontaneously secreting IgG and IgM. ${ }^{11}$ Leung et al suggested that Kawasaki disease may be caused by a superantigen, a staphylococcal or streptococcal toxin-producing expansion and stimulation of $\mathrm{V}$-beta 2 positive $T$ cells in peripheral blood in the acute phase of the illness and in a more recent paper showed V-beta 2 positive cells in CD4 and CD8 subsets of $T$ cells infiltrating the myocardium and coronary artery in a five month old child who died in the acute phase of the disease. ${ }^{12}{ }^{13}$ It has been postulated by several workers that defective immune responses to EBV may play a role in the pathogenesis of Kawasaki disease.

Recently, we performed a necropsy on a six year old child who had died suddenly while at play. His death was attributed to myocardial ischaemia due to widespread areas of stenosis in both coronary arteries and an acquired giant coronary aneurysm. Necropsy tissues were investigated for the presence of EBV infection in the light of growing evidence of a link between EBV, Kawasaki disease and coronary artery aneurysms.

\section{Case report}

A six year old caucasian boy collapsed while attending a children's birthday party. Resuscitation was unsuccessful and he died one hour after arrival in the Accident and Emergency Department. Over the previous week, the child had complained of an influenza-like illness for which no medical consultation was sought. A detailed discussion with the family general 


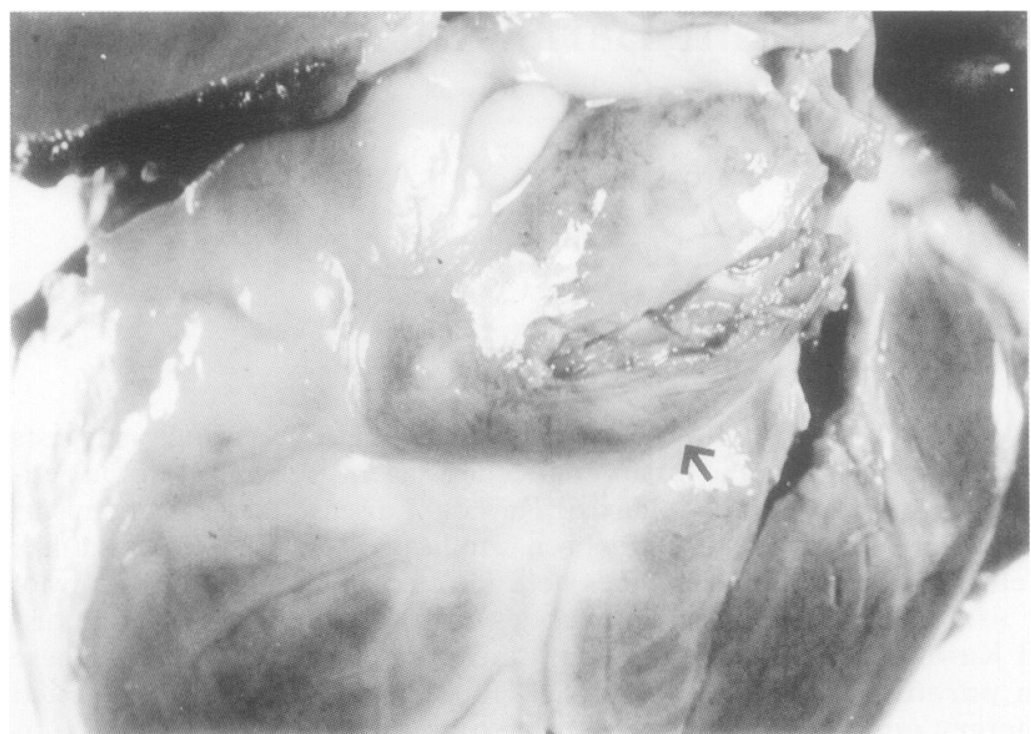

Figure 1 Frontal view of the heart showing a partially opened giant fusiform aneurysm of the left anterior descending coronary artery (arrow). The lumen is filled with a thrombus.

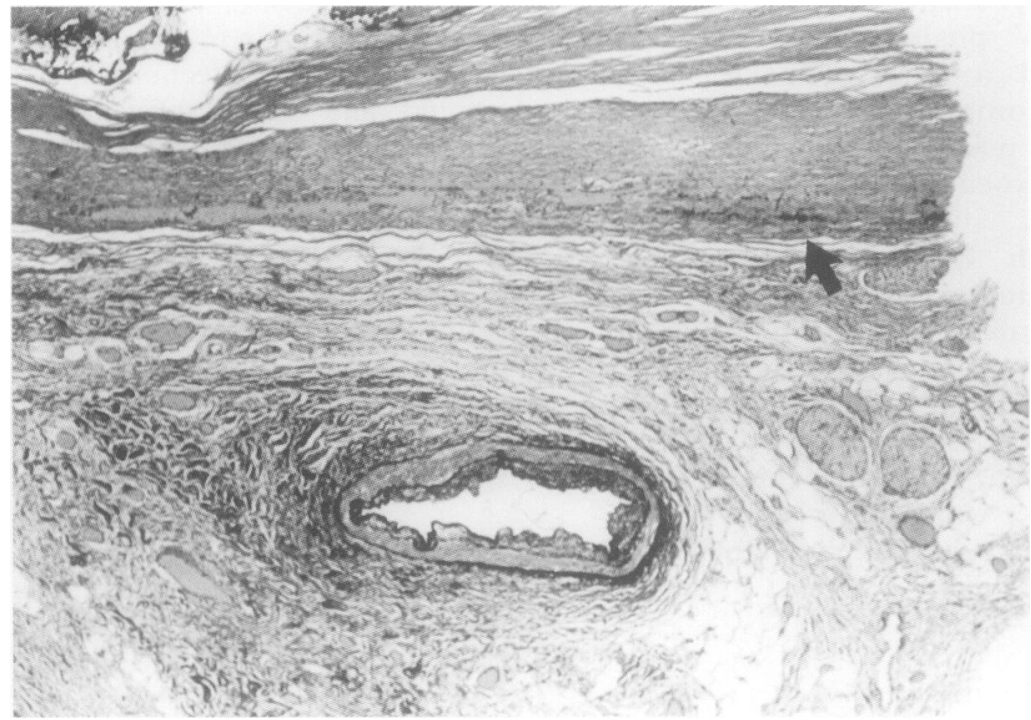

Figure 2 Elastic Van Gieson stained section of the left anterior descending coronary artery below the aneurysm filled with a partially calcified thrombus (arrow).

practitioner failed to show any serious previous illnesses, particularly with features of Kawasaki disease and the boy never displayed any cardiac symptoms.

At post mortem examination, there were no dysmorphic features or abnormal external findings. Examination of the lungs showed notable pulmonary oedema and congestion. The mucosa of the whole length of the trachea and bronchi was very congested and histology revealed a tracheobronchitis, possibly of initial viral aetiology with secondary bacterial infection with beta-haemolytic streptococcus Group A isolated from the bronchial and lung swabs. The heart was enlarged: $160 \mathrm{~g}$ (normal weight 90-100 g). The predominant left coronary artery showed a giant $(2 \mathrm{~cm}$ diameter) fusiform aneurysm $1 \mathrm{~cm}$ from the coronary ostium and immediately below the division into the left anterior descending and left circumflex branches (fig 1). This aneurysm was almost completely filled with thrombus and had a firm calcified wall. No lumen of the artery could be identified below the level of the aneurysmal dilation where the vessel showed a severe stenosis. Inspection of the right coronary artery showed this to be of a very small size with calcified thickened walls and notable stenosis of the lumen, but no evidence of aneurysmal dilation on macroscopic inspection. Histological examination of the walls of the right and left coronary arteries showed notable fibrosis of the media and intima with destruction of elastic lamina but no recognisable inflammatory infiltrates (fig 2). The myocardium showed only small foci of subendocardial fibrosis. The aorta and its main branches were normal and all other muscular arteries, in particular the renal arteries, had normal macroscopic appearances. Histological examination of peripheral arteries (renal and splenic) showed no evidence of vasculitis. Virological investigations of cerebrospinal fluid, trachea and lung tissue yielded negative results.

\section{In situ hybridisation}

Sections of paraffin wax embedded coronary artery and myocardium were hybridised with a biotinylated oligonucleotide probe with a nucleotide sequence complementary to EBVencoded RNA-1 (EBER-1), using a previously described method. An ABC (Dako, High Wycombe, UK) method incorporating nitroblue tetrazolium (NBT) as the chromogen was used to detect hybridisation. Control sections were hybridised with a nonsense probe with a base composition identical with that of EBER-1 probe. Positive controls consisted of an EBV positive case of Hodgkin disease and a case of post-transplantation lymphoma. No EBER-1 positive cells were identified in either the myocardium or walls of the coronary artery.

\section{Discussion}

The link between EBV, Kawasaki disease and coronary artery aneurysms is not a straightforward one. The literature indicates that approximately $50 \%$ of Kawasaki disease cases are EBV positive but that the presence of the virus is not a prerequisite for the disease. It is likely that in a proportion of children, an unusual response to the virus renders them more susceptible to vascular damage, probably through a deregulation of $\mathrm{T}$ helper cell activity. Kikuta et al suggested that Kawasaki disease may represent an unusual spectrum of a primary infection with EBV and showed in vivo seroconversion from seropositive for antibodies to EBV capsid antigens to completely seronegative to all EBV antigens. ${ }^{14}$ The same group of workers suggested that Kawasaki disease might recur through the reinfection of susceptible children or reactivation of latently infected lymphocytes after the first episode of Kawasaki disease. ${ }^{15}$ EBV genetic material was detected using PCR in peripheral blood mononuclear cells in $60 \%$ of patients with Kawasaki disease but only in $12 \%$ of control cases. ${ }^{16}$ In a chronic active form of EBV infection with coronary artery aneurysms, EBV DNA was detected in the heart of three patients and in the aorta of one. ${ }^{17}$ Purtilo et al reported the detection of the EBV genome using PCR methods in $76 \%$ of hearts, $60 \%$ of 
lymph nodes and in $82 \%$ of other tissues in necropsy cases of Kawasaki disease compared with negative results in 10 matched controls. Coronary artery smooth muscle cell nuclei expressed EBER-1 in five of six cases. ${ }^{18}$

Despite some increase in our understanding of the aetiology of Kawasaki disease, there are still no definitive laboratory investigations available for the diagnosis of this disorder and well defined clinical criteria need to be met before the diagnosis is made. However, there are several examples in the literature, including our case, which display initial unrecognised subclinical presentation of the disease. Even pathological features of the acute or chronic disease are not specific enough to allow the diagnosis to be made with certainty. The pathological features of an acute phase of Kawasaki disease resemble those seen in infantile polyarteritis nodosa with focal involvement of large to medium-sized arteries with acute necrotising arteritis, aneurysmal dilation and acute thrombosis. ${ }^{19}$ The early inflammatory reaction is confined to the intima, extending later transmurally with destruction of the media. Destruction of the elastic lamellae, intimal thickening, aneurysmal dilation, and thrombosis of the lumen are late vascular changes. It has been suggested that thickening of the intima seen in patients with Kawasaki disease resembles changes of atherosclerosis and the evolution of the lesions with regression of aneurysm is related to the initial severity of the arterial damage and treatment. ${ }^{20}$ The large aneurysms are easily found at necropsy, but the presence of stenoses underscores the importance of a detailed examination of the coronary vessels in all sudden unexpected deaths in young children. Atypical Kawasaki disease has to be considered in the differential diagnosis of giant aneurysms. Although EBV aetiology was not proven in our case, it is likely that in many cases an unusual immunological reaction to a primary EBV infection leads to severe coronary artery damage in early childhood.
1 Levin M, Tizard EJ, Dillon MJ. Kawasaki disease: recent advances. Arch Dis Child 1991;66:1369-74.

2 Koren G, Lavi S, Rose V, Rowe R. Kawasaki disease, a review of risk factors for coronary aneurysms. $\mathcal{F}$ Pediatr 1986;108:388-9.

3 Rowley AH, Gonzales-Crussi F, Shulman ST. Kawasaki syndrome. Rev Infect Dis 1988;10:1-15.

4 Durongpisitkul K, Gururaj VJ, Park JM, Martin CF. The prevention of coronary artery aneurysm in Kawasaki disease: a meta-analysis on the efficacy of aspirin and immunoglobulin treatment. Pediatrics 1995;96:1057-61.

5 Hsu CH, Chen MR, Hwang FY, Kao HA, Hung HY, Hsu $\mathrm{CH}$. Efficacy of plasmin-treated intravenous gammaglobulin for therapy of Kawasaki syndrome. Pediatr Infect Dis ₹ 1993;12:509-12.

6 Wreford FS, Conradi SE, Cohle SD, Lie JT, Dana SE, Puri $S$. Sudden death caused by coronary artery aneurysms: a late complication of Kawasaki disease. $\mathcal{F}$ Forensic Sci 1990; late comp

7 Hamashima Y, Kishi K, Tasaka K. Rickettsia-like bodies in infantile acute febrile mucocutaneous lymph node syndrome [letter]. Lancet 1973;ii:42.

8 Kegel SM, Dorsey TJ, Rowen M, Taylor WF. Cardiac death in mucocutaneous lymph node syndrome. Am 7 Cardiol 1977;40:282-6.

9 Yanagihara R, Todd JK. Acute febrile mucocutaneous lymph node syndrome. Am $\mathcal{F}$ Dis Child 1980;138:603-15.

10 Shulman ST, Rowley AH. Does Kawasaki disease have a retroviral aetiology? Lancet 1986;ii:545-6.

11 Leung DYM, Chu ET, Wood N, Grady S, Meade R, Geha RS. Immunoregulatory $\mathrm{T}$ cell abnormalities in mucocutaneous lymph node syndrome. F Immunol 1983;130:2002-4

12 Leung DYM, Meissner HC, Fulton DR, Murray DL Kotzin BL, Schlievert PM. Toxic shock syndrome toxinsecreting Staphylococcus aureus in Kawasaki syndrome. Lancet 1993;342:1385-8.

13 Leung DYM, Giorno RC, Kazemi LV, Flynn PA, Busse JB. Evidence for superantigen involvement in cardiovascular injury due to Kawasaki syndrome. F Immunol 1995;155: injury due

14 Kikuta $\mathrm{H}$, Mizuno F, Osato T. Kawasaki disease and an unusual primary infection with Epstein-Barr virus [letter]. Pediatrics 1984;73:413-14

15 Kikuta H, Matsumoto S, Yanase Y, Kawasaki T, Mizuno F, Osato T. Recurrence of Kawasaki disease and Epstein-Barr virus infection [letter]. F Infect Dis 1990;162:1215.

16 Kikuta H, Nakanishi M, Ishikawa N, Konno M, Matsumoto $S$. Detection of Epstein-Barr virus sequences in patients with Kawasaki disease by means of the polymerase chain reaction. Intervirology 1992;33:1-5.

17 Kikuta H, Sakiyama Y, Matsumoto S, Hamada I, Yazaki M, Iwaki $\mathrm{T}$, et al. Detection of Epstein-Barr virus DNA in cardiac and aortic tissues from chronic, active Epstein-Barr virus infection associated with Kawasaki disease-like coronary artery aneurysms. F Pediatr 1993;123:90-2.

18 Purtilo DT, Luka J, Patton H, Fujiwara H, Fujiwara T, England J, et al. EBV genome detection in tissues of patients with Kawasaki syndrome [abstract]. Lab Invest 1993;68: 106A.

19 Melish ME, Hicks RM. Mucocutaneous lymph node syndrome in the United States. Am $\mathcal{F}$ Dis Child 1976;130: 599-607.

20 Akagi T, Rose V, Benson LN, Newman A, Freedom RM Outcome of coronary artery aneurysms after Kawasaki disease. $\mathcal{F}$ Pediatr 1992;121:689-94. 